\title{
Whole-body MRI in pediatric patients with cancer
}

\author{
Marcos Duarte Guimarães ${ }^{1,2}$, Julia Noschang ${ }^{3 *}$, Sara Reis Teixeira ${ }^{4}$, Marcel Koenigkam Santos ${ }^{4}$, \\ Henrique Manoel Lederman ${ }^{5}$, Vivian Tostes ${ }^{6}$, Vikas Kundra ${ }^{7}$, Alex Dias Oliveira ${ }^{3}$, Bruno Hochhegger ${ }^{8}$ \\ and Edson Marchiori ${ }^{9}$
}

\begin{abstract}
Cancer is the leading cause of natural death in the pediatric populations of developed countries, yet cure rates are greater than $70 \%$ when a cancer is diagnosed in its early stages. Recent advances in magnetic resonance imaging methods have markedly improved diagnostic and therapeutic approaches, while avoiding the risks of ionizing radiation that are associated with most conventional radiological methods, such as computed tomography and positron emission tomography/computed tomography. The advent of whole-body magnetic resonance imaging in association with the development of metabolic- and function-based techniques has led to the use of whole-body magnetic resonance imaging for the screening, diagnosis, staging, response assessment, and post-therapeutic follow-up of children with solid sporadic tumours or those with related genetic syndromes. Here, the advantages, techniques, indications, and limitations of whole-body magnetic resonance imaging in the management of pediatric oncology patients are presented.
\end{abstract}

Keywords: Neoplasm, Pediatrics, Magnetic resonance imaging, Whole body MRI, Whole body imaging

\section{Background}

Cancer is currently the leading cause of natural death in the pediatric populations of developed countries [1]. However, the cure rates for cancers are greater than $70 \%$ in some cases when a cancer is diagnosed in its early stages. To increase the cure rates for cancer patients, diagnostic and therapeutic advances are needed. To select the most appropriate treatment for a child with cancer, the type, location, and staging of the tumour should be completely assessed [2-4]. Ideally, imaging protocols should be rapid, provide high quality images, have a low radiation, and provide clinically significant information [5-7]. In addition, every effort should be made to avoid redundant examinations that do not provide additional information relevant to therapeutic decision making [8]. There are many imaging modalities that are currently used to characterise the extent of local and distant disease. For example, ultrasonography, computed tomography (CT), magnetic resonance imaging (MRI),

\footnotetext{
*Correspondence: julia_noschang@hotmail.com

${ }^{3}$ Department of Imaging, AC Camargo Cancer Center, Rua Prof. Antônio

Prudente, 211, Liberdade, Sao Paulo/SP 01509-010, Brazil

Full list of author information is available at the end of the article
}

metaiodobenzylguanidine (MIBG) scans, and bone scintigraphy (BS) are most frequently performed $[8,9]$. However, modalities that deposit radiation, such as BS and CT, should be used with caution in pediatric patients due to the risk of complications, including the risk of developing secondary malignancies.

Positron emission tomography/computed tomography (PET/CT) has recently been used in children with cancer because it provides whole-body (WB) coverage and information regarding the metabolic stage of tumours. Regarding the latter, an intravenous injection of the radiopharmaceutical, ${ }^{18} \mathrm{~F}$-fluorodeoxyglucose $\left({ }^{18} \mathrm{~F}-\mathrm{FDG}\right)$, allows regions of abnormal glucose metabolism to be detected with hybrid systems such as PET/CT and correlated with possible morphological changes on anatomic images $[10,11]$. PET/CT also plays an important role in tumour staging, in assessing response to treatment, and can potentially predict treatment success in certain oncology settings [12]. Operational limitations of PET/CT include its restricted availability to specialised centers in many countries; the short half life of ${ }^{18} \mathrm{~F}$ which requires prompt delivery and use [13]; and the radiation burden to a patient. 
In contrast, MRI can provide exquisite anatomic detail and functional information without radiation to patients. In addition, technological advances, particularly in the development of fast imaging sequences, allow MRI to provide WB coverage in a reasonable time frame $[14,15]$. Thus, with the fusion of morphological sequences and functional techniques, such as diffusion-weighted imaging (DWI) and WB morphological/functional mapping, relevant information regarding disease activity can be obtained [16, 17]. The aim of this review is to discuss the advantages, techniques, indications, and limitations of WB MRI in evaluations of pediatric patients with cancer.

\section{Main Text}

\section{Advantages of WB MRI}

WB MRI provides a single examination of the entire body without the use of ionizing radiation. In addition to the excellent contrast and spatial resolution of WB MRI, functional information is obtained which improves the capacity of this method to differentiate normal

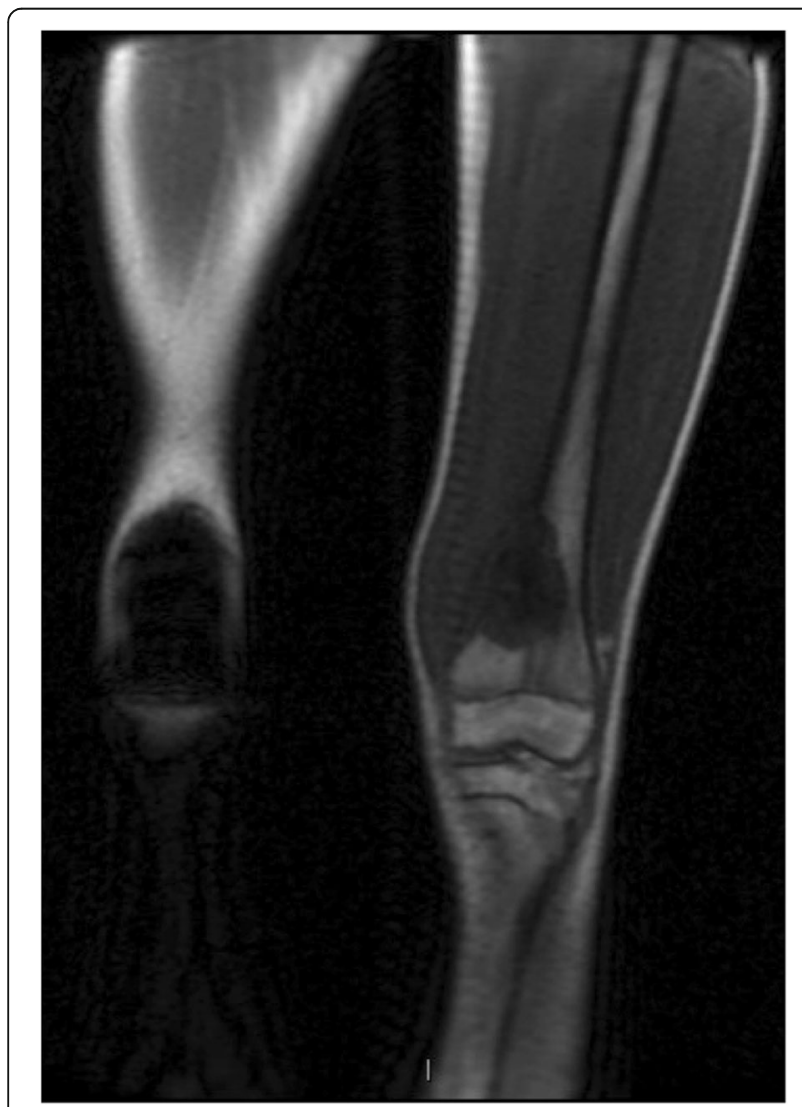

Fig. 1 A 15-year-old male patient follow up whole-body MRI examination by bilateral retinoblastoma and osteosarcoma in right femur, in the last exam, the T1-weighted image demonstrated a lesion with low signal intensity in distal left femur. Histological diagnosis of second osteosarcoma tissues from pathological tissues. MRI equipment is often available at both large and small centers, thereby facilitating the implementation of more advanced studies, such as MRI diffusion, perfusion, and WB studies $[18,19]$. Furthermore, a complete disease assessment in the oncology setting, including detection of metastasis sites, in a single examination helps to reduce the number of patient visits to an imaging service, thereby reducing related costs [20].

Exposure to ionizing radiation is a major concern in pediatric patients with cancer [7]. However, imaging methods that use ionizing radiation sources, such as $\mathrm{X}$ ray, $\mathrm{CT}, \mathrm{BS}$, and $\mathrm{PET} / \mathrm{CT}$, are often routinely employed $[21,22]$. The risk of tumour development due to ionizing radiation exposure is related to the amount, intensity, and accumulation of the applied radiation over an individual's life [23]. Newer CT scanners use iterative image reconstruction which reduces radiation exposure, although, exposure still occurs and repeated studies can incur a significant radiation dose for a patient. This risk increases when radiation exposure occurs at younger ages, especially exposure during childhood. In a recent study that examined the risks associated with the use of imaging methods that employ ionizing radiation for

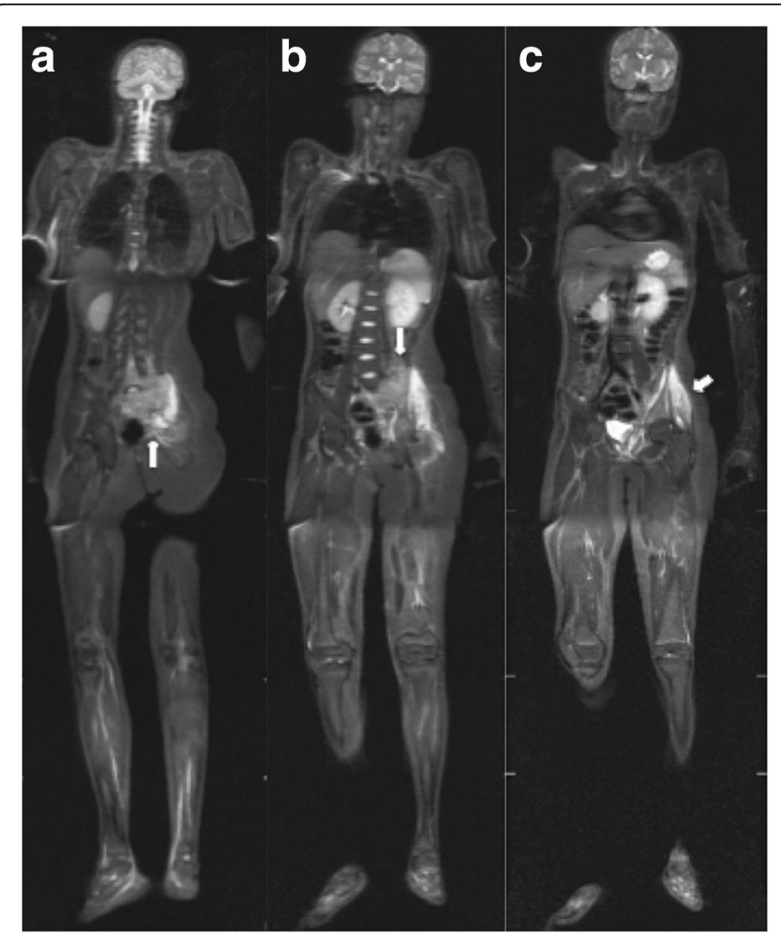

Fig. 2 An 18-year-old female patient with a voluminous lesion in the left hemi-pelvis (a, arrow) and histologically confirmed chondrosarcoma underwent whole-body MRI with a coronal STIR sequence for staging. The examination revealed involvement in the sacrum (a, arrow) and left ilium (b, arrow) and the presence of soft-tissue components adjacent to marked hyperintensity (c, arrow). Note the antalgic position, with slight body deviation to the right. No other lesion suspicious of malignancy was noted 


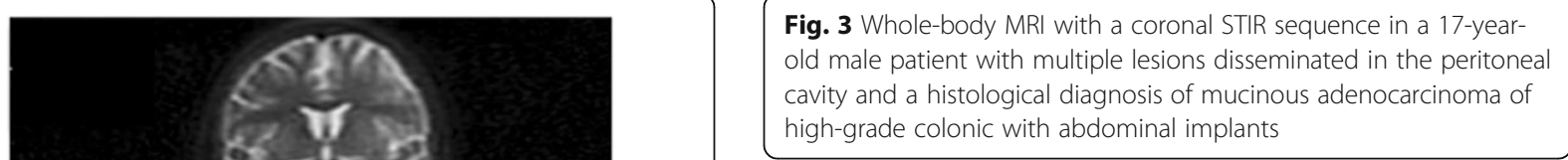

Fig. 3 Whole-body MRI with a coronal STIR sequence in a 17-yearold male patient with multiple lesions disseminated in the peritonea high-grade colonic with abdominal implants

diagnostic purposes, an estimated 29,000 new cancer cases were found to be related to the number of CT scans performed in the United States in 2007, with 15\% of the estimated cancers associated with scans that were performed on patients younger than 18 years $[24,25]$. An advantage of WB MRI is that it can be applied to atrisk populations, including those that may be affected by familial syndromes, to conduct cancer screenings. Advances in genetics have enabled the identification of patients with hereditary syndromes related to the development of neoplasms. The goal of performing WB MRI for cancer screenings is to detect malignancies in their early stages when the effectiveness of treatments and cure rates are optimal $[26,27]$. Ideally, screenings should be applied to apparently healthy populations that are at high risk for tumour development. Examples of inherited syndromes that are associated with increased risks of cancer include: multiple endocrine neoplasias I and II (e.g., endocrine tumours), Von Hippel-Lindau syndrome (e.g., renal carcinomas), familial adenomatous polyposis (e.g., colorectal tumours), and Li-Fraumeni syndrome (e.g., various types of tumours including sarcomas) (Fig. 1) [28-31]. Correspondingly, a new screening protocol that involves WB MRI has recently been proposed for patients with Li-Fraumeni syndrome [32].

\section{Magnetic resonance technique / protocol}

To date, there is no overall consensus regarding a WB MRI protocol for children. Typically, T1 and T2 imaging are performed with free-breathing, suspended respiration, or physiological motion control. It is also widely accepted that short tau inversion recovery (STIR) sequences and diffusion add diagnostic value to WB MRI examinations. Depending on a pediatric patient's age and size, a complete set of images are obtained in a single acquisition (e.g., for infants), or in two or more segmental acquisitions (e.g., for older children and adolescents). The images are subsequently aligned using specific software to enable visualization of the entire body [20,33]. WB MRI should be performed with highfield ( 21.5 Tesla) equipment with surface and/or body coils. Dedicated pediatric receiver coils are currently being introduced and will progressively have increased availability. Patients should be examined from head to toe in a supine position with their arms parallel to the body and their legs together. Coronal acquisition, which is more rapid than other approaches, is preferred, although at least one sequence (e.g., diffusion) should be acquired in the axial plane to compensate for the 


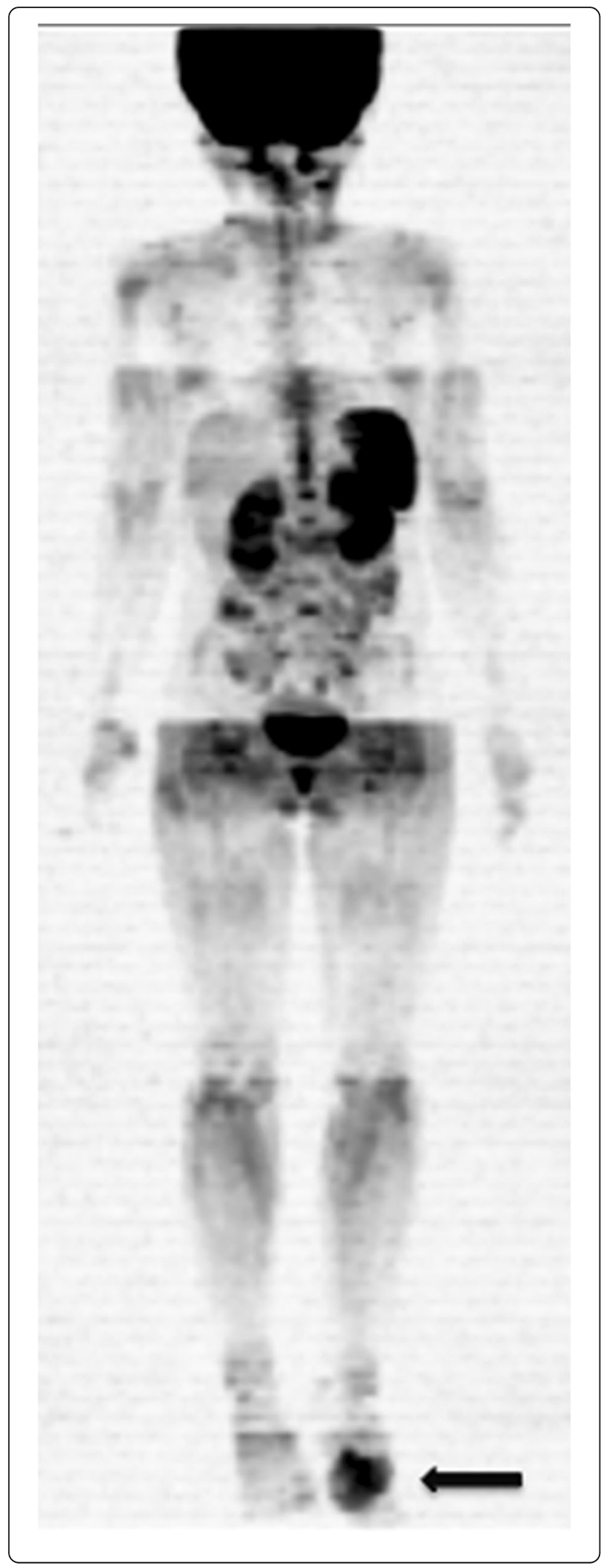

Fig. 4 A 9-year-old male patient with a histological diagnosis of osteosarcoma in the left foot (arrow) underwent whole-body MRI with diffusion sequences with three-dimensional reconstruction for staging, which showed no other lesion site

limitations associated with coronal acquisition (e.g., for the ribs, sternum, cranium, and spine) [34]. Sagittal acquisition is helpful for evaluating the spine. However, depending on the manufacturer, it is possible to perform axial T1-weighted acquisitions without wasting time. Moreover, synchronization of respiratory and cardiac movements can be achieved with external gates to avoid physiological motion artifacts [5, 33].

Conventional sequences, including $\mathrm{T} 1-$ and $\mathrm{T} 2$ weighted spin-echo sequences, are usually performed without the administration of a paramagnetic contrast medium. Malignant lesions are usually hypointense (low signal intensity) on T1-weighted images (Fig. 1) and have a high signal intensity on T2-weighted images [35].

The STIR sequence is highly sensitive for the detection of pathologic lesions. Bone marrow lesions, including marrow infiltration from lymphoma, metastases, and tumour-related edema, exhibit high signal intensity on STIR sequences (Figs. 2 and 3). Focal parenchymal lesions can be distinguished by their slightly different signal intensity in STIR sequences, while pathologic lymph nodes cannot be differentiated from normal nodes on the basis of signal intensity. The STIR technique also cannot be used to differentiate benign conditions from malignant neoplastic lesions. The latter limitation restricts the application of STIR in WB MRI in oncologic patients after treatment, since therapy-induced marrow changes, such as edema, necrosis, fibrosis, or red marrow hyperplasia, cannot be differentiated from viable tumours. However, STIR may be very useful in staging pediatric tumours; although, additional clinical experience and data are needed to determine its efficacy [36, 37].

Diffusion-weighted MRI sequences are increasingly being employed for WB evaluations of patients with cancer (Fig. 4). These sequences detect the random motion of water molecules, also known as Brownian motion, through biological tissues by detecting the protons in the water molecules. The movement of the water molecules causes a phasic dispersion of proton spin, thereby resulting in signal loss due to diffusion sensitivity. The signal intensity of an object of study is analyzed quantitatively by calculating the absolute apparent diffusion coefficient (ADC, in $\mathrm{mm}^{2} / \mathrm{s}$ ) of the object relative to the diffusion of water molecules in the proximal region $[16,38]$. This qualitative and quantitative analysis is primarily influenced by the presence of barriers that restrict the diffusion of water molecules in their microenvironment, and this produces imaging contrast between tissues. Thus, the signal 


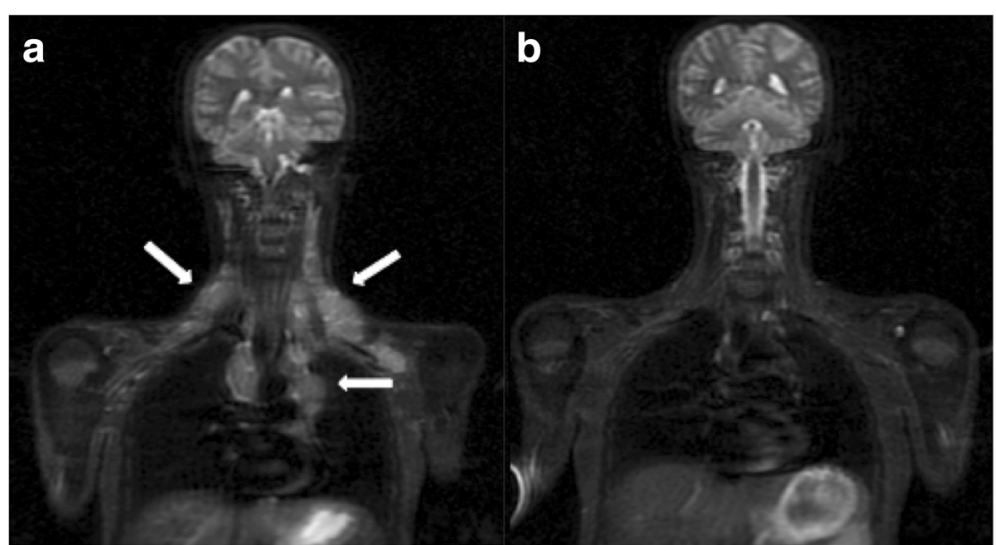

Fig. 5 Whole-body MRI with a coronal STIR sequence highlighting the cervical and thoracic regions of a17-year-old female patient with histologically confirmed non-Hodgkin lymphoma. a Multiple cervical lymphadenopathies in the supraclavicular and anterior mediastinal regions (arrows) were detected at diagnosis. b A follow-up examination performed 15 days after chemotherapy showed that the lesions had disappeared

intensities and ADCs of different tissues are distinct as a result of their structural characteristics. The restricted diffusion of water molecules that characterises malignant tumours is potentially due to the increased cell density of these tumours, thereby resulting in increased signal intensity in DWI and reduced ADCs, both of which facilitate detection of malignant lesions [16]. However, it should be noted that particularly in children, a high signal in DWI with body background suppression (DWIBS) can be a normal finding in the bony pelvis and lumbar spine. The different types of tissues with high cellularity that are present within these bones contribute to this high signal [39].

Currently, paramagnetic contrast agents can be used in WB MRI examinations, although they are not always indicated. It has been considered that the addition of post-contrast sequences significantly increases examination time and that the behavioral characteristics of lesion enhancement cannot be examined in the arterial, venous, and equilibrium phases simultaneously in WB scans, especially when multiple organs are involved [40]. However, strategies have been developed to address these limitations. For example, the Dixon technique achieves a uniform separation of water and fat that is resistant to large-field inhomogeneities compared to fat suppression by chemical shift selective saturation (CHESS) [41]. Furthermore, when properly implemented, the Dixon technique can be used to acquire either T1-weighted [42] or T2-weighted [43] images within a single breath hold. These rapid Dixon sequences have been successfully incorporated into a WB MRI protocol that is capable of providing multisequence and multiplanar scans, including triphasic (arterial, portal-venous, and equilibrium or delayed) contrastenhanced imaging of the liver, in approximately $1 \mathrm{~h}$ [44]. This Dixon-based WB MRI with multisequence and multiplanar images are also complementary and facilitate high-confidence reading, while multisequence and triphasic contrast-enhanced abdominal imaging is very useful for the detection and characterization of lesions in the liver, an imaging examination that is more commonly performed in adult examinations $[44,45]$. On the other hand, however, it is important to consider that administration of paramagnetic contrast agents are associated with some risks, such as those for accidental

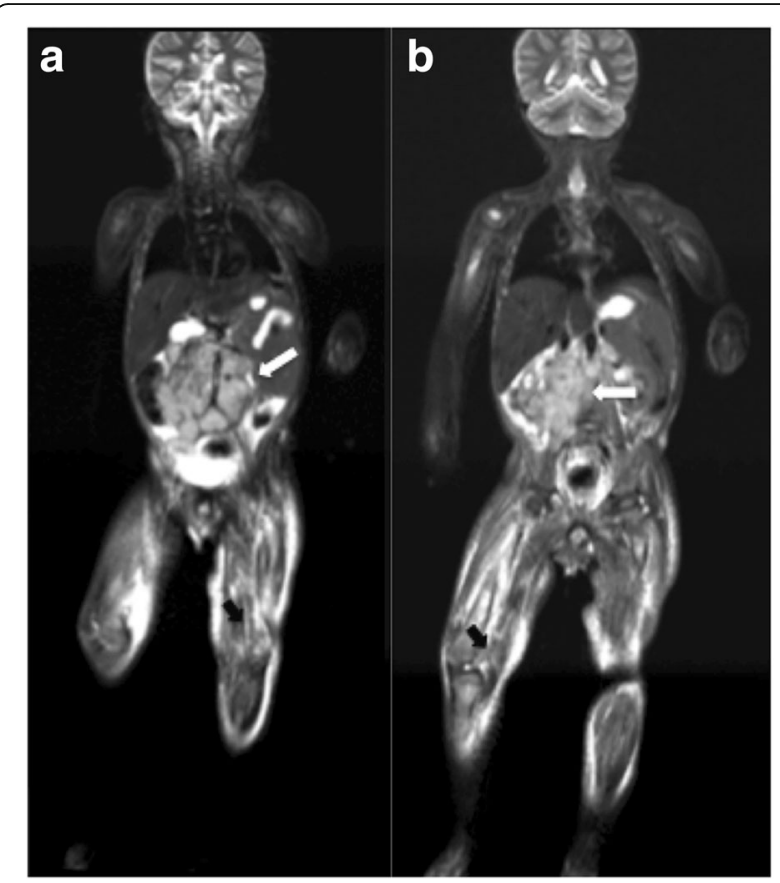

Fig. 6 Whole-body MRI with a coronal STIR sequence was performed in a 3-year-old male patient with histologically confirmed neuroblastoma. $\mathbf{a}$ and $\mathbf{b}$ The primary lesion appears as an extensive retroperitoneal mass (white arrows), and multiple bone metastases (black arrows) are present in the femoral 


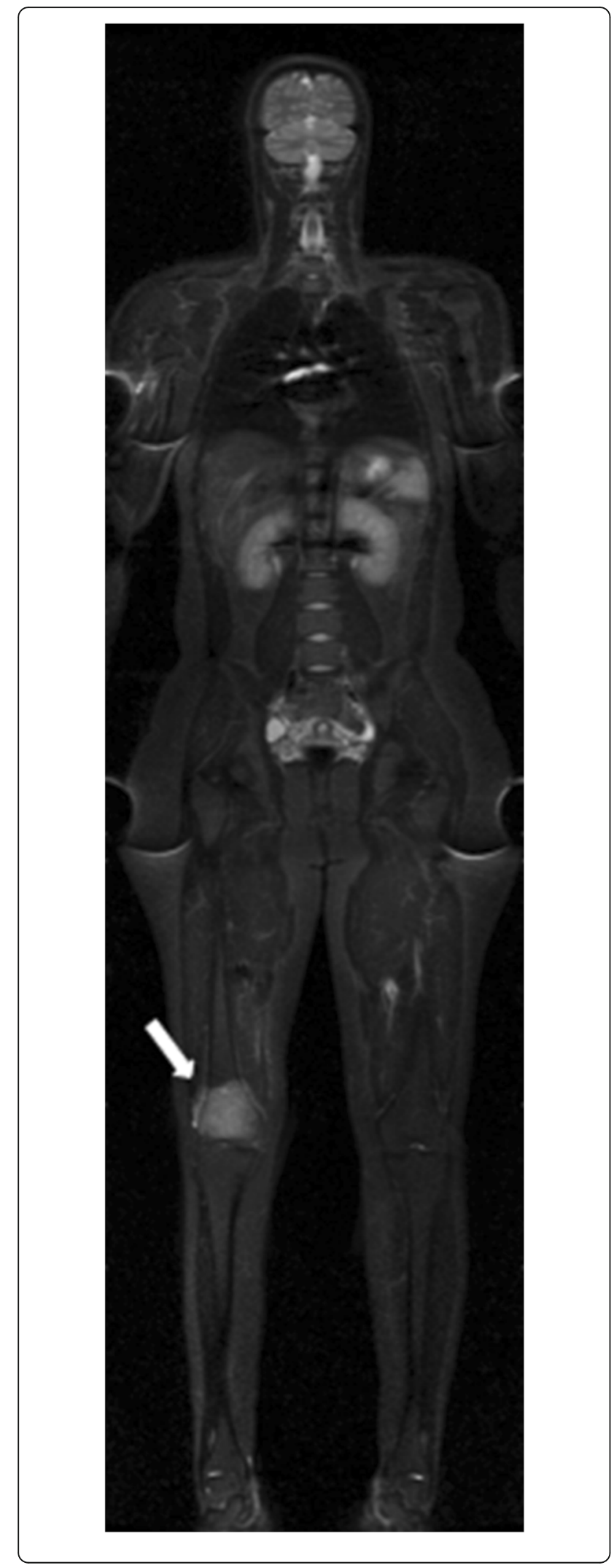

Fig. 7 Whole-body MRI with a coronal STIR sequence in a15-yearold male patient with a lesion in the right distal femur and a histological diagnosis of Ewing's sarcoma showed surrounding soft-tissue components (arrow), but no other area of abnormal signal intensity suggestive of malignancy

puncture, allergic reaction, renal failure, and nephrogenic systemic fibrosis [46]. Consequently, the prudent use of gadolinium-based contrast agents to avoid or minimise the risk of nephrogenic systemic fibrosis cannot be overemphasised, as pediatric oncologic patients are more likely to have impaired renal function secondary to anti-cancer therapy [47]. Pediatric patients should also be examined for hepatic lesions following the administration of paramagnetic contrast reagents, despite hepatic lesions being less frequent in children than in adults.

For patients who are unable to receive gadoliniumbased contrast agents, ferumoxytol may be a useful MRI contrast agent. Ferumoxytol is an ultrasmall superparamagnetic iron oxide (USPIO) that is comprised of iron oxide particles surrounded by a carbohydrate coat. Initially, this agent was used to treat anemia in patients with chronic renal failure [48]. However, more recently, ferumoxytol has been investigated as an intravenous contrast agent in MRI. The advantages of ferumoxytol include its ability to be administered as a bolus injection, allergic and idiosyncratic reactions with its administration have been limited, and it is not associated with a risk for nephrogenic systemic fibrosis $[48,49]$. Femuroxytol also has a long intravascular half-life of 14-15 h [50], and thus, can be used to obtain different types of images. However, there is currently a paucity of data available regarding its use as a paramagnetic contrast agent.

\section{Clinical indications}

WB MRI can be applied for lesion detection/staging, evaluation of treatment response, and follow-up and screening of children with cancer predisposition syndromes.

\section{Lesion detection / staging}

Clinical indications for WB MRI in pediatric patients with cancer depend on the disease type and stage of management. For several types of neoplasms, WB MRI has been shown to be a valid alternative to CT, PET/CT, and scintigraphic studies $[20,35,47]$. Many studies have also shown that WB MRI can be applied at different times during cancer management, including during the screening, staging, response evaluation, and posttherapeutic follow-up stages [5, 20].

The capacity for WB MRI to detect lesions depends on several factors, including the anatomic site, size, 
histological type, and differentiation grade of the lesions being examined. WB MRI has exhibited good diagnostic accuracy in the staging of a variety of tumours, including both lymphomas and solid tumours [51]. In fact, staging of these neoplasms contributed to the development of this examination technique $[5,51]$. Currently, WB MRI can detect lesions present in various anatomical sites, such as the brain, cervical region, thoracic organs, abdomen, bone marrow, and musculoskeletal system. The performance of WB MRI has also been shown to be similar to that of PET/CT in the staging of different cancers, and superior to CT, BS, and scintigraphy with gallium in evaluations of certain osseous and extra-osseous metastases [35, 52, 53].

WB MRI enables a proper assessment of WB bone marrow and the detection of compromised neoplastic sites, including primary tumours and metastases arising from diffusion [37]. However, since normal red marrow impedes diffusion, this may confound disease detection in younger children. Patients with melanoma and Langerhans cell histiocytosis may be evaluated with WB MRI. In fact, the sensitivity, specificity, and accuracy of this method in these cases has been found to similar or superior to those of other methods, including those employing MIBG [20, 54]. WB MRI has also been shown to perform well in the detection of bone metastases, with a higher positive predictive value (94 vs. $76 \%$, respectively) and greater sensitivity ( 99 vs. $26 \%$, respectively) observed compared with bone scintigraphy [35]. The use of WB MRI is limited in the detection of rib and skull lesions, although the use of respiratory synchronization (triggering) has been shown to reduce the occurrence of motion artifacts, thereby improving the ability of WB MRI to evaluate these anatomic sites $[20,55]$.

MRI provides different image contrasts that represent specific tissue characteristics. This is important for evaluations of primary tumours and metastases in the brain. Furthermore, if a lesion is detected in the brain or in another part of the body during a WB MRI exam, then a region-specific exam also needs to be conducted. Thus, for patients with Li-Fraumeni syndrome who have an elevated risk of brain tumours, a brain-specific protocol should be added to a WB MRI protocol.

Oncologic patients may undergo multiple MRI, thereby receiving repeated administrations of a gadolinium-based contrast agent. A high signal in the dentate nucleus and globus pallidus on unenhanced T1-weighted images should be cautiously evaluated in these patients, since the signal observed may be a consequence of the number of times that a gadolinium-based contrast material was administered, and not due to the presence of pathologic lesions $[56,57]$.

Currently, the most important clinical applications of WB MRI in children include the staging of malignant disease and screening for metastatic spread. These applications are particularly relevant in cases involving lymphoma and solid tumours.

\section{Lymphoma}

Diagnostic imaging provides important information regarding the staging and response assessment of lymphomas. Recently, a combination of CT and PET was applied to lymphoma staging and evaluations of treatment response [58]. However, both PET and CT involve substantial radiation exposure, and children often undergo

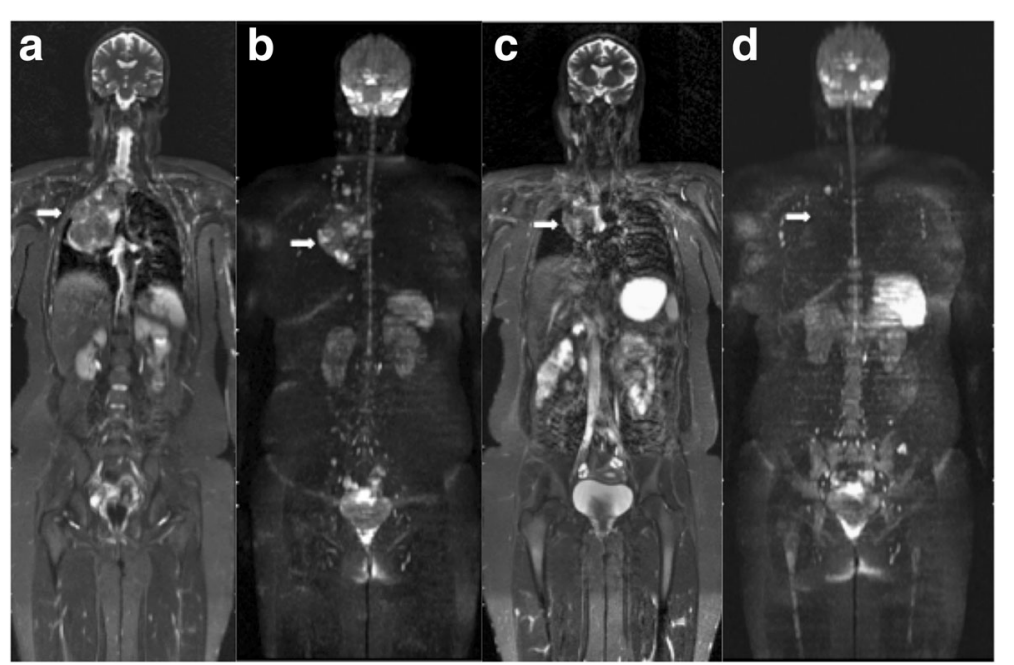

Fig. 8 A 16-year-old female patient with primary mediastinal Hodgkin lymphoma (stage III-B) underwent whole-body MRI with STIR (a, c) and diffusion (b, d) sequences. a and $\mathbf{b}$ Examinations performed for pre-therapeutic staging showed a voluminous anterior mediastinal lesion (arrows). c and $\mathbf{d}$ Post-therapeutic imaging showed the presence of a residual mediastinal lesion with no sign of activity (arrows) 
several PET/CT examinations during a treatment course. Thus, WB MRI represents a radiation-free alternative for lymphoma staging and follow-up (Fig. 5). Furthermore, when WB MRI and CT were compared in their capacity to provide staging of lymphoma, WB MRI was able to provide disease staging, detect lymph nodes greater than $1.2 \mathrm{~cm}$ (with sensitivity and specificity values of 92.0 and $99.9 \%$, respectively), and evaluate the presence or absence of disease spread to bone marrow [58]. In a study of eight children with lymphoma, WB MRI with a coronal STIR sequence was also more sensitive than conventional imaging (e.g., radiography, nuclear medicine studies - bone scintigraphy and gallium

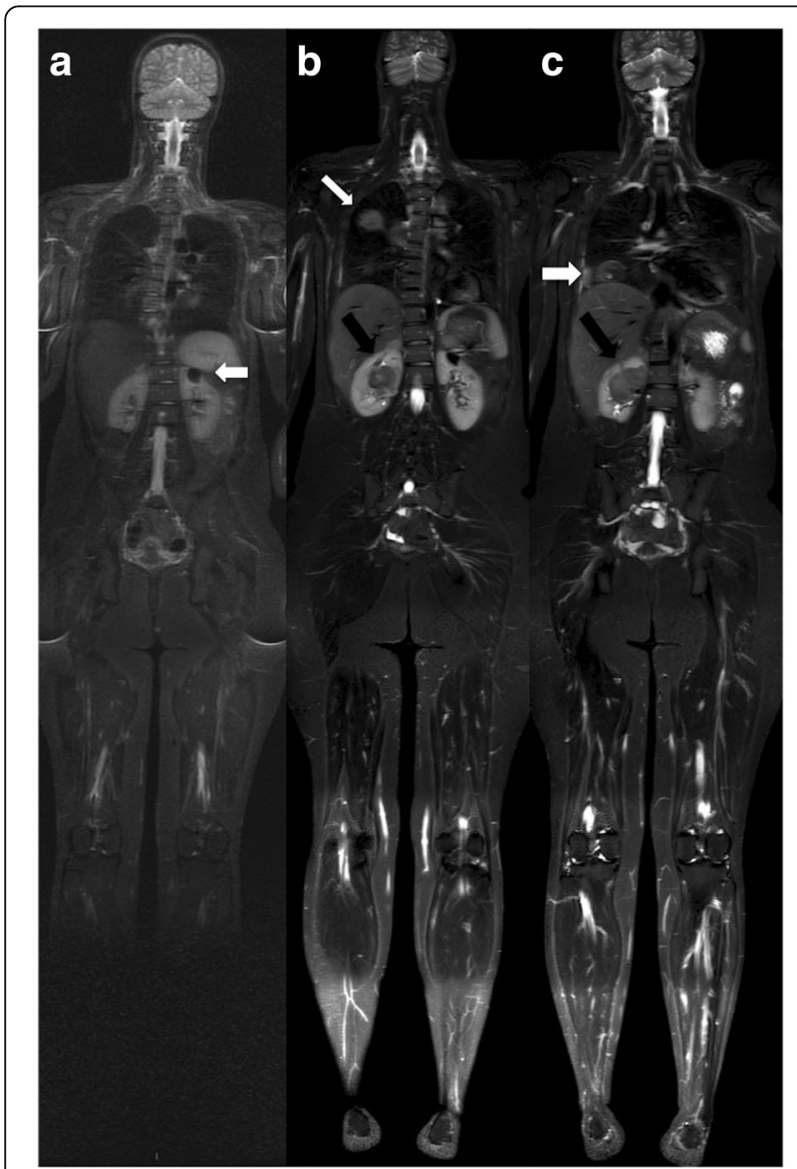

Fig. 9 A 20-year-old female patient with Li-Fraumeni syndrome in whom multiple neoplasms had developed since childhood, including lymphoma, soft-tissue sarcomas in the back and thigh, malignant fibrous histiocytoma in the buttock, and adrenal carcinoma. Follow up whole-body MRI examination since 2013, in the last the coronal STIR sequence demonstrated the presence that new lung lesions and kidney nodule. Histological diagnosis suggestive that metastasis of pleomorphic undifferentiated sarcoma in lung and renal cell carcinoma in kidney. Current Whole-body MRI with a coronal STIR sequence demonstrate the presence of multiple lesions in lung $(\mathbf{b}, \mathbf{c}$ white arrows) and the kidney nodule (b, c black arrows), compared to previous exam which demonstrated only the presence of simple renal cyst (arrow), with no other change suggestive of malignancy (a) scintigraphy - and CT) in detecting bone marrow involvement in the initial stages of disease [15]. Following treatment, however, residual and therapy-induced bone marrow signal abnormalities could not be differentiated from lymphomatous involvement [15].

\section{Solid tumours}

In various pediatric studies, the sensitivity of WB MRI for the detection of distant metastases has been compared with the sensitivities of radiography, CT, conventional MRI, nuclear medicine studies, and PET/CT [53, 54, 59]. Additional studies have suggested that WB MRI is a promising method for the detection of metastases in patients with small cell tumours, and that WB MRI provides at least equivalent information to conventional

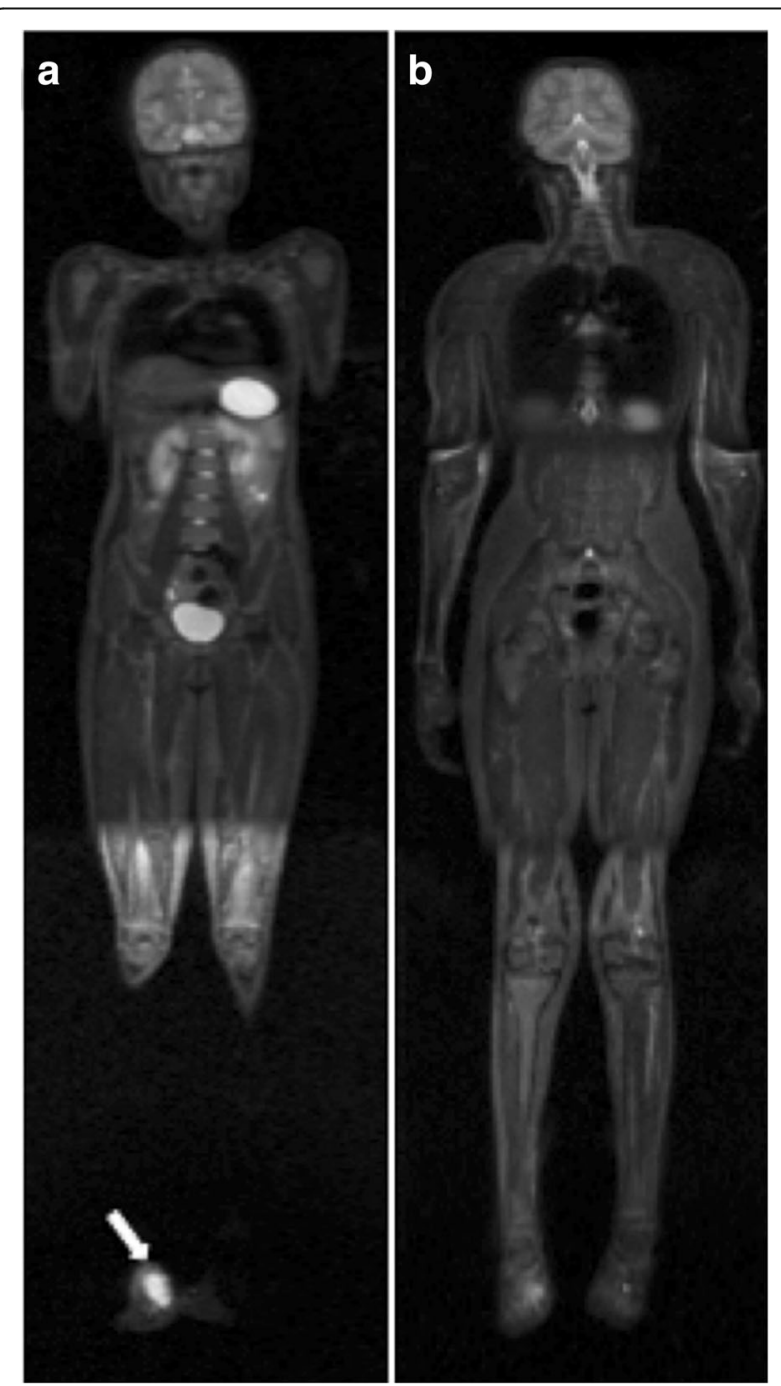

Fig. 10 A 16-year-old male patient with histologically confirmed osteosarcoma on the dorsum of the right foot underwent whole-body MRI with a coronal STIR sequence for staging. a Note the lesion in the right foot (arrow). b No skip metastasis or distant lesion was detected 
imaging studies [53, 59]. Neuroblastoma (Fig. 6), primitive neuroectodermal tumour, rhabdomyosarcoma, and Ewing's sarcoma (Fig. 7) are small round-cell malignancies that have been found to occur in the pediatric population. For the detection of metastases to bone, most investigators have reported a sensitivity of more than 97\% for WB MRI, and WB MRI has consistently exhibited a sensitivity comparable to, or greater than, that of skeletal scintigraphy with technetium $99 \mathrm{~m}\left({ }^{99 \mathrm{~m}} \mathrm{Tc}\right)$ medronate disodium $[35,53,59]$.

\section{Evaluation of treatment response and follow-up}

WB MRI can be used to evaluate therapeutic response in pediatric oncology patients $[51,60,61]$, and the information obtained can be used in combination with Response Evaluation Criteria in Solid Tumors (RECIST) [61]. For example, WB MRI can provide a morphological assessment of target lesions by measuring their major axes according to RECIST, while also providing a functional evaluation of lesions with diffusion sequences. Several studies have described the use of WB MRI to identify partial or complete responses, including increased absolute ADC, after the application of chemo- or radiotherapy to brain tumours, liver tumours, and sarcomas [62, 63]. Similar to CT and PET/CT, WB MRI can also be useful in evaluating significant morphological and functional improvements in lymphomas (Fig. 8), and these are often characterised by an inverse correlation between the tendency toward increased ADC and reduced tumour volume $[35,52]$. Furthermore, WB MRI can help distinguish between abnormal scarring and recurrence after therapy [64], thereby enabling the detection of any complications that are related or unrelated to disease or treatment.

\section{Screening of children with cancer predisposition syndromes}

Cancer predisposition syndromes include a multitude of cancers in which a mode of familial inheritance has been clearly established, although a specific genetic defect may not have been identified [65]. WB MRI has been useful in the screenings of children with cancer predisposition syndromes, and it also has the potential to provide a preclinical diagnosis of any associated tumours. For example, at some institutions, WB MRI is performed annually to screen for tumours in children with $\mathrm{Li}$ -

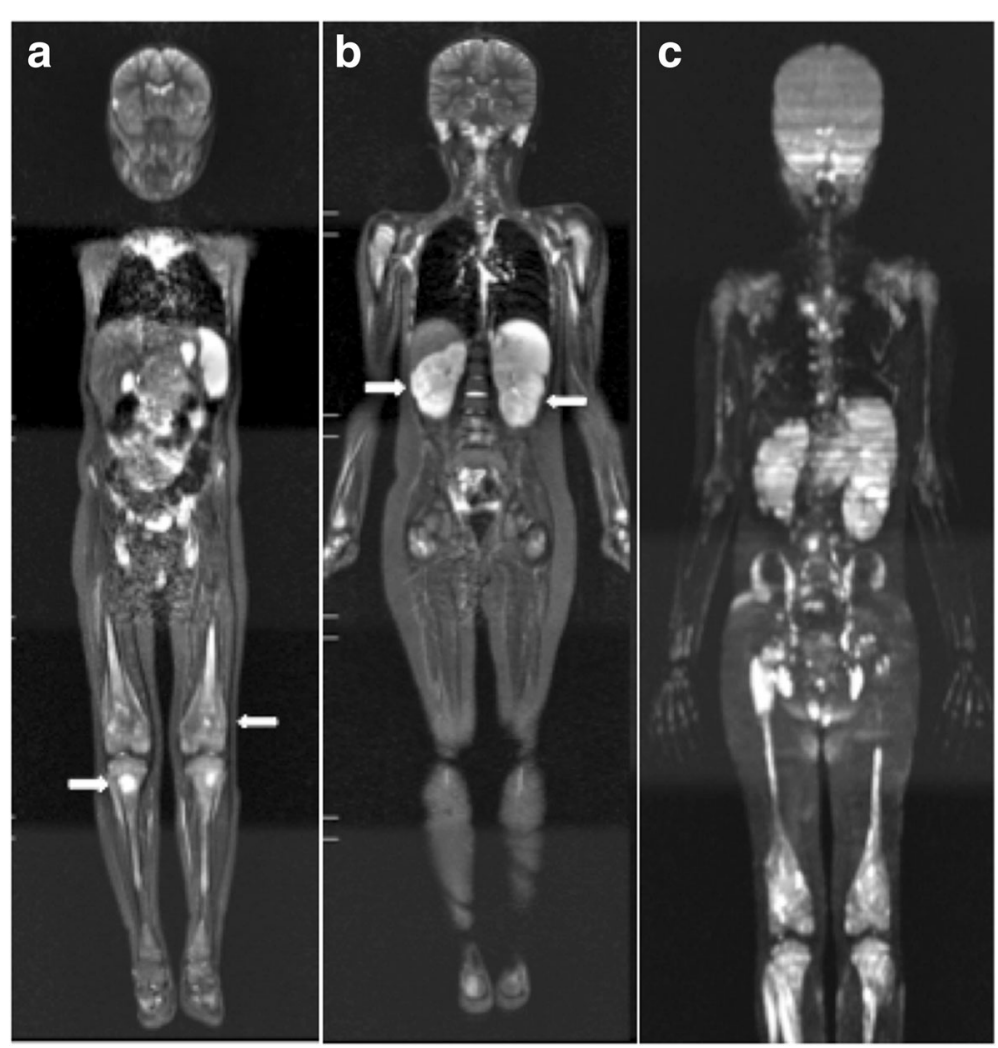

Fig. 11 Whole-body MRI was performed in a 6-year-old male patient with acute lymphoblastic leukemia for staging. a Diaphyseal and metaphyseal lesions in the distal femoral and proximal tibial regions are represented by hyperintense signals in a coronal STIR sequence (arrows). b Signs of bilateral renal infiltration by the underlying pathology (arrows) were observed. c A diffusion sequence demonstrated multiple hyperintense foci, consistent with leukemic infiltration 
Fraumeni syndrome (Fig. 9). Li-Fraumeni syndrome is an autosomal dominant hereditary syndrome that is caused by a loss-of-function mutation in the TP53 gene and affected individuals have a lifelong increased risk of osteosarcoma (Fig. 10), soft-tissue sarcoma, leukemia (Fig. 11), breast cancer, brain tumour, melanoma, and adrenal cortical tumours [65]. Similarly, individuals with hereditary retinoblastoma (RB) have a very high risk of developing subsequent malignant neoplasms, with osteosarcoma being the most common. When WB MRI screening tests were performed for survivors of hereditary RB, the sensitivity and specificity of detecting subsequent malignant neoplasms was 66.7 and $92.1 \%$, respectively [66].

\section{Limitations}

The use of WB MRI in pediatric oncological clinical practice is limited in some cases. For example, standard contraindications to conventional MRI, such as the presence of metallic body implants or history of claustrophobia, can also preclude the use of WB MRI. Due to the examination time of WB MRI (which can range from $30 \mathrm{~min}$ to $1 \mathrm{~h}$ ), the use of sedation or general anesthesia is typically needed in a significant proportion of pediatric patients, especially those who are young or uncooperative. Thus, the risks associated with these agents must be considered. Immobilization during the examination is also essential for preventing motion artifacts which can impair image acquisition and interpretation of the findings. In addition, physiological artifacts related to respiratory movements, heartbeat, and intestinal peristalsis can impair image acquisition [67]. To minimise the occurrence of these artifacts, multi-channel equipment, body coils, and parallel imaging can be employed. Furthermore, single-shot acquisition, the use of presaturation bands in the anterior body, and mechanisms of synchronization with respiratory and cardiac movements can reduce the time needed for an examination and facilitate the acquisition of highquality images $[67,68]$. Finally, the occurrence of false-positive results is another limiting factor in the use of WB MRI. For example, inflammatory abnormalities, infections, and even benign lesions such as simple cysts or vascular lesions have been found to simulate malignant lesions [67].

\section{Conclusion}

Currently, WB MRI is able to provide total body coverage, high tissue contrast, and good spatial resolution without the use of radiation. Moreover, the ability to obtain relevant morphological and functional information in a single examination represents a key advantage of this method in the management of pediatric oncology patients.

\begin{abstract}
Abbreviation
${ }^{18}$ F-FDG: ${ }^{18}$ F-fluorodeoxyglucose; ${ }^{99 m}$ Tc: Technetium 99m; ADC: Absolute apparent diffusion coefficient; ADC: Absolute apparent diffusion coefficient; BS: Bone scintigraphy; CHESS: Chemical shift selective saturation; CT: Computed tomography; DWI: Diffusion- weighted imaging; DWIBS: Diffusion-weighted imaging with body background suppression; MIBG: Metaiodobenzylguanidine; MRI: Magnetic resonance imaging; PET/CT: Positron emission tomography/ computed tomography; RB: Retinoblastoma; RECIST: Response Evaluation Criteria in Solid Tumors; STIR: Short tau inversion recovery; USPIO: Ultrasmall superparamagnetic iron oxide; WB: Whole body
\end{abstract}

\section{Acknowledgements \\ None. \\ Funding \\ Not applicable. \\ Availability of data and materials \\ Not applicable.}

Authors' contributions

MDG conceived of the topic for the review and the methodology used in this work. MDG, JN, SRT, MKS, HML VST, ADO, EM, BH undertook the literature search and manuscript drafting. MDG, VK controlled and checked the manuscript. All authors have read, edited and approved the final manuscript.

\section{Competing interests}

The authors declare that they have no competing interests.

\section{Consent for publication}

Not applicable.

Ethics approval and consent to participate

Not applicable.

\section{Author details}

${ }^{1}$ Department of Imaging, AC Camargo Cancer Center, Rua Prof. Antônio Prudente, 211, Liberdade, São Paulo/SP 01509-010, Brazil. Universidade Federal do Vale do São Francisco (UNIVASF), Av. José de Sá Maniçoba, Petrolina, PE 56304-917, Brazil. ${ }^{3}$ Department of Imaging, AC Camargo Cancer Center, Rua Prof. Antônio Prudente, 211, Liberdade, Sao Paulo/SP 01509-010, Brazil. ${ }^{4}$ Division of Radiology, Department of Internal Medicine, Ribeirao Preto Medical School, University of Sao Paulo, Av. Bandeirantes, 3900, Ribeirao Preto/ SP 14049-090, Brazil. ${ }^{5}$ Universidade Federal de São Paulo,

Departamento de Diagnóstico Por Imagem, Disciplina de Diagnóstico por Imagem em Pediatria, Rua Napoleão de Barros, 800, Vila Clementino, Sao Paulo/SP 04024002, Brazil. ' Universidade Federal de São Paulo, Centro de Diagnóstico por Imagem do Instituto de Oncologia Pediátrica e Médica Radiologista do Centro de Diagnóstico por Imagem do Instituto de Oncologia Pediátrica, Rua Napoleão de Barros, 800, Vila Clementino, Sao Paulo/SP 04024002, Brazil. 'Department of Diagnostic Radiology, The University of Texas MD Anderson Cancer Center, 1515 Holcombe Blvd, Houston, TX 77030, USA. ${ }^{8}$ Department of Radiology, Universidade Federal de Ciências da Saúde de Porto Alegre, Rua Professor Anes Dias, 285, Centro Histórico, Porto Alegre/RS 90020-090, Brazil. ${ }^{9}$ Department of Radiology, Universidade Federal do Rio de Janeiro, Rua Thomaz Cameron, 438, Valparaíso, Petrópolis/RJ 25685-129, Brazil.

Received: 30 September 2016 Accepted: 18 January 2017

Published online: 10 February 2017

\section{References}

1. Chatenoud L, Bertuccio P, Bosetti C, Levi F, Negri E, La Vecchia C. Childhood cancer mortality in America, Asia and Oceania, 1970 through 2007. Cancer. 2010;116(21):5063-74.

2. Kleis M, Daldrup-Link H, Matthay K, Goldsby R, Lu Y, Schuster T, et al. Diagnostic value of PET/CT for the staging and restaging of pediatric tumors. Eur J Nucl Med Mol Imaging. 2009;36(1):23-36. 
3. Federico SM, Spunt SL, Krasin MJ, Billup CA, Wu J, Shulkin B, et al. Comparison of PET-CT and conventional imaging in staging pediatric rhabdomyosarcoma. Pediatr Blood Cancer. 2013;60(7):1128-34.

4. Hernandez-Pampaloni M, Takalkar A, Yu JQ, Zhuang H, Alavi A. F-18 FDGPET imaging and correlation with CT in staging and follow-up of pediatric lymphomas. Pediatr Radiol. 2006;36(6):524-31.

5. Ley S, Ley-Zaporozhan J, Schenk JP. Whole-body MRI in the pediatric patient. Eur J Radiol. 2009;70(3):442-51.

6. Schmidt GP, Haug A, Reiser MF, Rist C. Whole-body MRI and FDG-PET/CT imaging diagnostics in oncology. Radiologe. 2010;50(4):329-38.

7. Alzen G, Benz-Bohm G. Radiation protection in pediatric radiology. Dtsch Arztebl Int. 2011;108(24):407-14.

8. Oltmann SC, Garcia NM, Barber R, Hicks B, Fischer AC. Pediatric ovarian malignancies: how efficacious are current staging practices? J Pediatr Surg. 2010:45(6):1096-102.

9. Shapiro NL, Bhattacharyya N. Staging and survival for sinus cancer in the pediatric population. Int J Pediatr Otorhinolaryngol. 2009;73(11):1568-71.

10. Miller E, Metser U, Avrahami G, Dvir R, Valdmam D, Sira LB, et al. Role of 18F-FDG PET/CT in staging and follow-up of lymphoma in pediatric and young adult patients. J Comput Assist Tomogr. 2006;30(4):689-94.

11. Völker T, Denecke T, Steffen I, Misch D, Schönberger S, Plotkin M, et al. Positron emission tomography for staging of pediatric sarcoma patients: results of a prospective multicenter trial. J Clin Oncol. 2007;25(34):5435-41.

12. von Falck C, Maecker B, Schirg E, Boerner AR, Knapp WH, Klein C, et al. Post transplant lymphoproliferative disease in pediatric solid organ transplant patients: a possible role for [18F]-FDG-PET(/CT) in initial staging and therapy monitoring. Eur J Radiol. 2007:63(3):427-35.

13. Truong MT, Erasmus JJ, Macapinlac HA, Marom EM, Mawlawi O, Gladish GW, et al. Integrated positron emission tomography/computed tomography in patients with non-small cell lung cancer: normal variants and pitfalls. J Comput Assist Tomogr. 2005;29(2):205-9.

14. Kavanagh E, Smith C, Eustace S. Whole-body turbo STIR MR imaging: controversies and avenues for development. Eur Radiol. 2003;13(9): 2196-205.

15. Kellenberger CJ, Miller SF, Khan M, Gilday DL, Weitzman S, Bayn PS. Initial experience with FSE STIR whole-body MR imaging for staging lymphoma in children. Eur Radiol. 2004;14(10):1829-41.

16. Eiber M, Dütsch S, Gaa J, Fauser C, Rummeny EJ, Holzapfel K. Diffusionweighted magnetic resonance imaging (DWI-MRI): a new method to differentiate between malignant and benign cervical lymph nodes. Laryngorhinootologie. 2008;87(12):850-5.

17. Koh DM, Padhani AR. Diffusion-weighted MRI: a new functional clinical technique for tumour imaging. Br J Radiol. 2006;79(944):633-5.

18. Blomqvist L, Torkzad MR. Whole-body imaging with MRI or PET/CT: the future for single-modality imaging in oncology? JAMA. 2003;290(24):3248-9.

19. Chen W, Jian W, Li HT, Li C, Zhang YK, Xie B, et al. Whole-body diffusionweighted imaging vs. FDG-PET for the detection of non small-cell lung cancer. How do they measure up? Magn Reson Imaging. 2010;28(5):613-20.

20. Goo HW. Regional and whole-body imaging in pediatric oncology. Pediatr Radiol. 2011:41(1):S186-94.

21. Hall EJ, Brenner DJ. Cancer risks from diagnostic radiology. Br J Radiol. 2008; 81(965):362-78.

22. Davies $\mathrm{HE}$, Wathen $\mathrm{CG}$, Gleeson FV. The risks of radiation exposure related to diagnostic imaging and how to minimise them. BMJ. 2011;342:d947.

23. Brenner DJ, Shuryak I, Einstein AJ. Impact of reduced patient life expectancy on potential cancer risks from radiologic imaging. Radiology. 2011;261(1):193-8.

24. Berrington de Gonzalez A, Mahesh M, Kin KP, Bhargavan M, Lewis R, Mettler F, et al. Projected cancer risks from computed tomographic scans performed in the United States in 2007. Arch Intern Med. 2009;169(22):2071-7.

25. Chawla SC, Federman N, Zhang D, Nagata K, Nuthakki S, McNitt-Gray M, et al. Estimated cumulative radiation dose from PET/CT in children with malignancies: a 5-year retrospective review. Pediatr Radiol. 2010;40(5):681-6.

26. Egger M, Zwahlen M. Tumor screening. Ther Umsch. 2013;70:193-4.

27. Ducreux M, Mateus C, Planchard D, Fizazi K. Screening and early diagnosis of other cancers (non-small cell lung carcinoma, urologic cancers, liver cancer and melanoma). Rev Prat. 2010;60(2):219-23.

28. Sweed MF, Vig HS. Hereditary colorectal cancer syndromes. Start risk assessment in primary care. Adv Nurse Pract. 2007;15(7):49-52.

29. Digweed M. Human genetic instability syndromes: single gene defects with increased risk of cancer. Toxicol Lett. 1993:67(1-3):259-81.

30. Malkin D. Li-fraumeni syndrome. Genes Cancer. 2011;2(4):475-84.
31. Testa JR, Malkin D, Schiffman JD. Connecting molecular pathways to hereditary cancer risk syndromes. Am Soc Clin Oncol Educ Book. 2013;33:81-90.

32. Villani A, Tabori U, Schiffman J, Schilien A, Beyene J, Druker $\mathrm{H}$, et al. Biochemical and imaging surveillance in germline TP53 mutation carriers with Li-Fraumeni syndrome: a prospective observational study. Lancet Oncol. 2011;12(6):559-67.

33. Schaefer JF, Kramer U. Whole-body MRI in children and juveniles. Rofo. 2011;183(1):24-36.

34. Koh DM, Collins DJ. Diffusion-weighted MRI in the body: applications and challenges in oncology. AJR Am J Roentgenol. 2007;188(6):1622-35.

35. Goo HW, Choi SH, Ghim T, Moon HN, Seo JJ. Whole-body MRI of paediatric malignant tumours: comparison with conventional oncological imaging methods. Pediatr Radiol. 2005;35(8):766-73.

36. Kellenberger CJ, Epelman M, Miller SF, Babyn PS. Fast STIR whole-body MR imaging in children. Radiographics. 2004;24(5):1317-30.

37. Karmazyn B, Cohen MD, Jennings SG, Robertson KA. Marrow signal changes observed in follow-up whole-body MRI studies in children and young adults with neurofibromatosis type 1 treated with imatinib mesylate (Gleevec) for plexiform neurofibromas. Pediatr Radiol. 2012; 42(10):1218-22

38. Takahara T, Imai Y, Yamashita T, Yasuda S, Nasu S, Van Cauteren M. Diffusion weighted whole body imaging with background body signal suppression (DWIBS): technical improvement using free breathing, STIR and high resolution 3D display. Radiat Med. 2004;22(4):275-82.

39. Ording Müller LS, Avenarius D, Olsen ØE. High signal in bone marrow at diffusion-weighted imaging with body background suppression (DWIBS) in healthy children. Pediatr Radiol. 2011;41(2):221-6.

40. Hasebroock KM, Serkova NJ. Toxicity of MRI and CT contrast agents. Expert Opin Drug Metab Toxicol. 2009;5(4):403-16.

41. Glover GH, Schneider E. Three-point Dixon technique for true water/fat decomposition with B0 inhomogeneity correction. Magn Reson Med. 1991; 18(2):371-83.

42. Ma J, Vu AT, Son JB, Choi H, Hazle JD. Fat-suppressed three-dimensional dual echo Dixon technique for contrast agent enhanced MRI. J Magn Reson Imaging. 2006;23(1):36-41.

43. Ma J, Son JB, Zhou Y, Le-Petross H, Choi H. Fast spin-echo triple-echo dixon (fTED) technique for efficient T2-weighted water and fat imaging. Magn Reson Med. 2007;58(1):103-9.

44. Ma J, Costelloe CM, Madewell JE, Hortobagyi GN, Green MC, Cao G, et al. Fast dixon-based multisequence and multiplanar MRI for whole-body detection of cancer metastases. J Magn Reson Imaging. 2009;29(5):1154-62.

45. Costelloe CM, Kundra V, Ma J, Chasen BA, Rohren EM, Bassett RL, et al. Fast Dixon whole-body MRI for detecting distant cancer metastasis: a preliminary clinical study. J Magn Reson Imaging. 2012;35(2):399-408.

46. Juluru K, Vogel-Claussen J, Macura K, Kamel IR, Steever A, Bluemke DA. MR imaging in patients at risk for developing nephrogenic systemic fibrosis: protocols, practices, and imaging techniques to maximize patient safety. Radiographics. 2009;29(1):9-22

47. Siegel MJ, Acharyya S, Hoffer FA, Wyly JB, Friedmann AM, Snyder BS, et al. Whole-body MR imaging for staging of malignant tumors in pediatric patients: results of the American College of Radiology Imaging Network 6660 Trial. Radiology. 2013;266(2):599-609.

48. Bashir MR, Bhatti L, Marin D, Nelson RC. Emerging Applications for Ferumoxytol as a Contrast Agent in MRI. J Magn Reson Imaging. 2015;41(4):884-98.

49. Weinstein JS, Varallyay CG, Dosa E, Gahramanov S, Hamilton B, Rooney WD, et al. Superparamagnetic iron oxide nanoparticles: diagnostic magnetic resonance imaging and potential therapeutic applications in neurooncology and central nervous system inflammatory pathologies, a review. J Cereb Blood Flow Metab. 2010;30(1):15-35.

50. McCullough BJ, Kolokythas O, Maki JH, Green DE. Ferumoxytol in Clinical Practice: Implications for MRI. J Magn Reson Imaging. 2013;37(6):1476-9.

51. Darge K, Jaramillo D, Siegel MJ. Whole-body MRI in children: current status and future applications. Eur J Radiol. 2008;68(2):289-98.

52. Punwani S, Taylor SA, Bainbridge A, Prakah V, Bandula S, De Vita E, et al. Pediatric and adolescent lymphoma: comparison of whole-body STIR halfFourier RARE MR imaging with an enhanced PET/CT reference for initial staging. Radiology. 2010;255(1):182-90.

53. Kumar J, Seith A, Kumar A, Sharma R, Bakhshi S, Kumar R, et al. Wholebody MR imaging with the use of parallel imaging for detection of skeletal metastases in pediatric patients with small-cell neoplasms: 
comparison with skeletal scintigraphy and FDG PET/CT. Pediatr Radiol. 2008;38(9):953-62.

54. Krohmer S, Sorge I, Krausse A, Kluge R, Bierbach U, Marwede D, et al. Whole-body MRI for primary evaluation of malignant disease in children. Eur J Radiol. 2010;74(1):256-61.

55. Goo HW. State-of-the-art pediatric chest imaging. Pediatr Radiol. 2013;43(3):261.

56. Roberts DR, Holden KR. Progressive increase of $\mathrm{T} 1$ signal intensity in the dentate nucleus and globus pallidus on unenhanced T1-weighted MR images in the pediatric brain exposed to multiple doses of gadolinium contrast. Brain Dev. 2016;38(3):331-6.

57. Kanda T, Ishii K, Kawagichi H, Kitajima K, Takenaka D. High signal intensity in the dentate nucleus and globus pallidus on unenhanced T1-weighted MR images: relationship with increasing cumulative dose of a gadoliniumbased contrast material. Radiology. 2014;270(3):834-41.

58. Brennan DD, Gleeson T, Coate LE, Cronin C, Carney D, Eustace SJ. A comparison of whole-body MRI and CT for the staging of lymphoma. AJR Am J Roentgenol. 2005;85(3):711-6.

59. Mazundar A, Siegel MJ, Narra V, Luchtman-Jones L. Whole-body fast inversion recovery MR imaging of small cell neoplasms in pediatric patients: a pilot study. AJR Am J Roentgenol. 2002;179(5):1261-6.

60. Li SP, Padhani AR. Tumor response assessments with diffusion and perfusion MRI. J Magn Reson Imaging. 2012;35(4):745-63.

61. Padhani AR, Koh DM. Diffusion MR imaging for monitoring of treatment response. Magn Reson Imaging Clin N Am. 2011;19(1):181-209.

62. Padhani AR, Khan AA. Diffusion-weighted (DW) and dynamic contrastenhanced (DCE) magnetic resonance imaging (MRI) for monitoring anticancer therapy. Target Oncol. 2010;5(1):39-52.

63. Padhani AR, Liu G, Koh DM, Chenevert TL, Thoeny HC, Takanara T, et al. Diffusion-weighted magnetic resonance imaging as a cancer biomarker: consensus and recommendations. Neoplasia. 2009;11(2):102-25.

64. Herman M, Paucek B, Raida L, Myslivecek M, Zapletalová J. Comparison of magnetic resonance imaging and (67)gallium scintigraphy in the evaluation of posttherapeutic residual mediastinal mass in the patients with Hodgkin's lymphoma. Eur J Radiol. 2007;64(3):432-8.

65. Monsalve J, Kapur J, Malkin D, Babyn PS. Imaging of cancer predisposition syndromes in children. Radio Graphics. 2011;31(1):263-80.

66. Friedman DN, Lis E, Sklar CH, Oeffinger KC, Reppucci M, Fleischut MH, et al. Whole-body magnetic resonance imaging (WB-MRI) as surveillance for subsequent malignancies in survivors of hereditary retinoblastoma: a pilot study. Pediatr Blood Cancer. 2014;61 (8):1440-4

67. Koh DM, Blackledge M, Padhani AR, Takahara T, Kwee TC, Leach MO, et al. Whole-body diffusion-weighted MRl: tips, tricks, and pitfalls. AJR Am J Roentgenol. 2012;199(2):252-62.

68. Koh DM, Takahara T, Imai Y, Collins DJ. Practical aspects of assessing tumors using clinical diffusion-weighted imaging in the body. Magn Reson Med Sci. 2007;6(4):211-24.

\section{Submit your next manuscript to BioMed Centra and we will help you at every step:}

- We accept pre-submission inquiries

- Our selector tool helps you to find the most relevant journal

- We provide round the clock customer support

- Convenient online submission

- Thorough peer review

- Inclusion in PubMed and all major indexing services

- Maximum visibility for your research

Submit your manuscript at www.biomedcentral.com/submit

) Biomed Central 